\title{
Evolução do Processo de Carbonatação em Argamassas de Cal Aérea
}

\author{
Evolution of Carbonation Process \\ in Aerial Lime Mortars
}

\author{
Mateus Antônio Nogueira Oliveira ${ }^{1}$, Miguel Ângelo Dias Azenha ${ }^{2}$, \\ Paulo José Brandão Barbosa Lourenço ${ }^{2}$, José Victor Brasil de Souza ${ }^{3}$
}

\footnotetext{
${ }^{1}$ Universidade Federal de Minas Gerais, Departamento de Engenharia de Estruturas, Escola de Engenharia, Campus Pampulha, CEP: 31270-901, Belo Horizonte, Minas Gerais, Brasil.

${ }^{2}$ Universidade do Minho, Escola de Engenharia, ISISE, CEP: 4804-533, Guimarães, Portugal.

${ }^{3}$ Universidade Federal do Pará, ITEC, Campus Guamá, CEP: 66075-110, Belém, Pará, Brasil. e-mail: mateusengcivil@gmail.com, josevictorbrasi1002@gmail.com,miguel.azenha@civil.uminho.pt; pbl@civil.uminho.pt
}

\section{RESUMO}

A cal aérea vem sendo utilizada como aglomerante durante séculos e diferentes tipos de argamassas foram fabricadas por todo mundo usando o material durante a história da humanidade. Diversas propriedades, por exemplo químicas e mecânicas, dessas argamassas estão associadas ao processo de carbonatação, neste sentido um conjunto de experimentos são propostos para estudar o avanço do referido fenômeno. A pesquisa foi inicializada com a definição da composição de argamassa: 1: 1,3: 3 (cal: água: agregado), a definição seguiu composições de referências bibliográficas bem como um processo iterativo. A influência do tamanho dos corpos-de-prova na evolução da carbonatação foi avaliada adotando-se cilindros com cinco diâmetros diferentes, que foram testados com fenolftaleína em três diferentes idades (10, 21 e 90 dias). Discos com espessura reduzida $(\sim 2 \mathrm{~mm})$ foram armazenados em três ambientes (padrão, com elevada umidade e elevado $\mathrm{CO}_{2}$ ), amostras foram coletadas e testadas por análise termogravimétrica em diferentes idades $(1,4,7,14,21,28$ dias). Os resultados indicam uma significativa influência dos tamanhos dos espécimes na profundidade da carbonatação e no ambiente na evolução da carbonatação, com variações de profundidade carbonatada de $\sim 120 \%$ sendo que espécimes com dimensões reduzidas, quando avaliados com fenolftaleína, apresentaram reação mais rápida, bem os como corpos-de-provas armazenadas no ambiente com alto $\mathrm{CO}_{2}$. Nos dois casos, a umidade elevada na fase inicial tende a reduzir a velocidade da reação.

Palavras-chave: Cal aérea, argamassa, carbonatação, análise termogravimétrica, fenolftaleína

\begin{abstract}
Aerial lime has been used as binder for centuries and different types of mortars were fabricated using the material during the history around the world. Different properties of the aerial lime mortars are associated with the carbonation process (e.g. chemical, mechanical response), in this sense a set of experiments are proposed to study the progress of the mentioned phenomenon. The research was initialized with the definition of the mortar composition: 1:1.3:3 (lime:water:aggregate). This definition followed an iterative process considering the consistence of the mixture and the bibliographical references. The influence of the size of specimens on the carbonation evolution was evaluated adopting cylinders with five different diameters. They were tested using phenolphthalein spray at three different ages (10, 21 and 90 days). Discs with reduced thickness $(\sim 2 \mathrm{~mm})$ were stored in three different environments (standard, high humidity and high $\mathrm{CO}_{2}$ concentration), samples were collected at different aged $(1,4,7,14,21,28$ days) and tested by thermogravimetric analysis (TGA). The results indicate a significant effect of the size of specimens on the carbonation depth and the environment on the evolution of carbonation, with depth variation of $\sim 120 \%$, specimens with reduced dimensions when evaluated with phenolphthalein spray presented a faster reaction as well as the small specimens store in the environment with high $\mathrm{CO}_{2}$. In both cases the elevated humidity on the initial phase tends to reduce the velocity of the reaction.
\end{abstract}

Keywords: Aerial lime, mortar, carbonation, thermogravimetric analysis, phenolphthalein. 


\section{INTRODUÇÃO}

As estruturas históricas de alvenaria têm importantes aspectos sociais, arqueológicos, estéticos, econômicos, políticos, arquitetônicos e técnicos que devem ser considerado [1]. Neste sentido, a preservação de construções históricas é de significativa importância para a sociedade e para as gerações futuras [2]. Considerandose esses fatos, a conservação e estudo de estruturas antigas é uma tarefa complexa e que requer uma análise aprofundada com detalhes que vão além da análise estrutural simples [1,2].

Uma grande variedade de argamassas foi usada na construção de monumentos históricos e outras estruturas [1-4]. Numerosas civilizações antigas (Fenícios, Gregos, romanos e outros) utilizaram a cal aérea na preparação de argamassas [1-4]. No século XIX, com o aparecimento do cimento Portland houve uma queda considerável na utilização dos materiais à base de cal, tanto hidráulica quanto aérea [1]. Existem diferentes formas de classificação de cal aérea, referências sobre a referida temática podem ser obtidas na literatura [5, 6], esse ligante atualmente tem sido utilizado em diferentes formas, mas especialmente no reparo de alvenarias antigas, uma vez que o cimento Portland mostra baixa afinidade físico-química [7] e distintas propriedades mecânicas, sendo muitas vezes prejudicial [8-10].

Propriedades típicas de argamassas feitas à base de cal aérea incluem alta permeabilidade, flexibilidade e plasticidade, tendência a retrair em estágios iniciais de endurecimento, solubilidade em água e baixa resistência mecânica [1,11].

Em termos de alvenarias históricas, muitas que existem ao longo de todo o mundo estão em estado vulnerável, nesse sentido a conservação estrutural e/ou aplicações de reforço são necessárias [1, 11, 12].

As estruturas históricas com argamassa à base de cal aérea possuem ainda mais a complexidade, porque as propriedades de material mudam significativamente ao longo do tempo devido ao processo de carbonatação $[1,11]$. Nesse processo, vários mecanismos estão envolvidos e acoplados durante a carbonatação [1, $11,13,14]$. Para quantificar a extensão e a evolução desse fenômeno é essencial entender os aspectos físicos, químicos e propriedades mecânicas da argamassa e consequentemente o desempenho da estrutura $[1,11,15]$. A carbonatação total da argamassa de uma alvenaria pode levar séculos a completar-se [16-18].

Em um contexto diferente, o uso de cal aérea para novas construções está alinhado com as modernas construção eco-eficiente [19]. Pelas considerações mencionadas, o estudo sobre argamassas de cal aérea, em especial o fenômeno da carbonatação é importante não só para o restauro de estruturas antigas, mas para um melhor conhecimento de aspectos estruturais, além de uma contextualização da construção moderna.

O presente trabalho pretende continuar com resultados experimentais para o melhor entendimento da evolução da carbonatação. objetivando-se a avaliação da segurança estrutural de construções antigas em alvenaria com foco nas estruturas com argamassa baseadas em cal aérea, bem como no estudo do endurecimento dessas argamassas.

\section{Considerações iniciais: Processo de carbonatação}

Apresenta-se nesse item informações sobre o processo de carbonatação das argamassas de cal aérea. Dada a elevada espessura, a carbonatação completa da argamassa de uma alvenaria espessa pode levar séculos [14, 20,21]. Nas estruturas à base de argamassas de cal aérea, a carbonatação é de fundamental importância para o endurecimento $[15,21]$. Como consequência do endurecimento diferencial da argamassa associado à evolução da frente de carbonatação, ocorrem ao longo dos anos modificações da distribuição interna de tensões nos elementos estruturais $[1,11]$. Diferentes casos de ruína ou danos das estruturas históricas podem ser vistas na literatura $[1,22,23]$.

Ao longo do tempo há uma variação dos compostos químico da argamassa, os componentes presentes na argamassa estão ilustrativamente representados na Figura 1. 


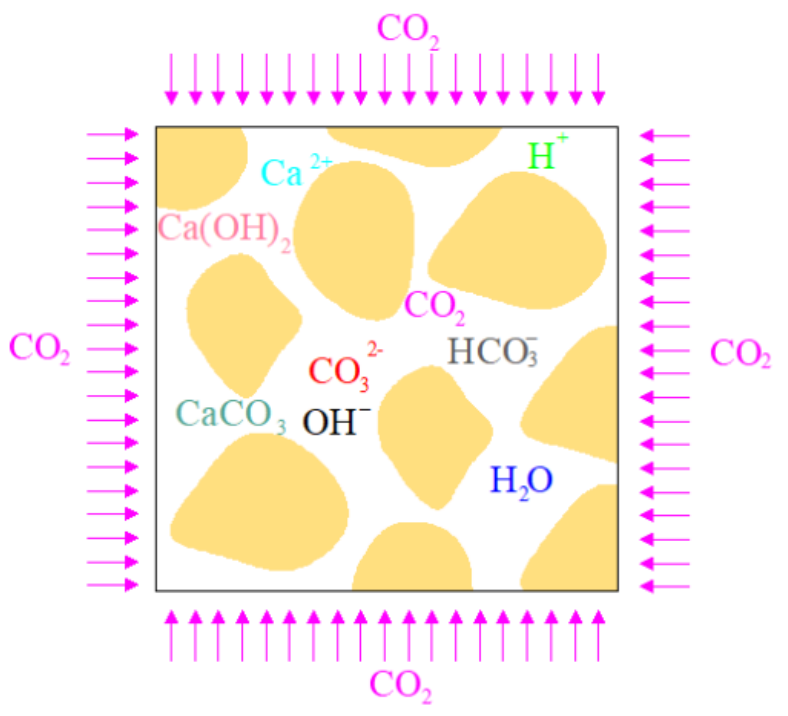

Figura 1: Componentes presentes na argamassa (adaptado de OLIVEIRA [1])

Durante o processo de carbonatação, existem cinco estágios principais envolvidos [1, 11, 24-26]:

1. difusão do $\mathrm{CO}_{2}$ através dos poros da argamassa;

2. dissolução do $\mathrm{CO}_{2}$ na água presente dos poros;

$$
\mathrm{Ca}(\mathrm{OH})_{2} \rightarrow \mathrm{Ca}^{2+}+2 \mathrm{OH}^{-}
$$

3. dissolução do $\mathrm{Ca}(\mathrm{OH}) 2$ na água dos poros;

$$
\mathrm{CO}_{2}+\mathrm{OH}^{-} \rightarrow \mathrm{HCO}_{3}^{-}
$$

4. equilíbrio químico do $\mathrm{CO}_{2}$ dissolvido na água dos poros;

$$
\mathrm{HCO}_{3}^{-}+\mathrm{OH}^{-} \rightarrow \mathrm{CO}_{3}^{-2}+\mathrm{H}_{2} \mathrm{O}
$$

5. precipitação do $\mathrm{CaCO}_{3}$.

A taxa de carbonatação depende de diversos fatores, com a concentração de $\mathrm{CO}_{2}$, a pressão do ar, o teor de umidade do calcário, a umidade ambiente, temperatura entre outros [1, 11, 15, 27-34].

O processo de carbonatação afeta diretamente características da argamassa, uma vez que a microestrutura do material é alterada com a precipitação do $\mathrm{CaCO}_{3}$, como mostrado acima. Fatores com a porosidade, a permeabilidade e a difussibilidade de fluidos são diretamente afetadas durante a evolução do fenômeno [9, 35-39]. A distribuição do tamanho dos poros também sofre alterações [24, 40], uma análise detalhada de diferentes trabalhos no que tange às modificações pelo processo de carbonatação pode ser visualizada em OLIVEIRA [1] .

No tocante a correlação entre a carbonatação e propriedades mecânicas, diferentes conclusões foram obtidas, mas todos indicam o aumento da resistência e do módulo de elasticidades [3, 12, 21, 24, 41-45]. No que tange a modelagem matemática da carbonatação, modelos de diferentes complexidade são apresentados na literatura, uma revisão detalhada de modelos multi-físicos para a avaliação da carbonatação pode ser obtida em OLIVEIRA [1].

\section{Métodos de medição da carbonatação}

Dada a relevância do fenômeno nessa seção, apresenta-se informações sobre diferentes métodos de medição da carbonatação. Os métodos para medição da carbonatação podem ser classificados em qualitativos e quantitativo $[1,15,21]$. Devido ao grande número de possibilidades, apenas dois métodos serão abordados nesse trabalho, a fenolfteleína e a análise termogravimétrica (TGA), sendo esses os métodos utilizados aqui. Outros métodos de medição da carbonatação são citados na literatura, por exemplo em LAWRENCE [46], LO e LEE [47], SLEGERS e ROUXHET [48] e VILLAIN et al. [49].

A fenolftaleína é um método tradicional para se detectar carbonatação, em resumo é feita a pulverização na superfície recém-quebrada [24, 46, 47, 50-55]. A recomendação apresentada pela RILEM [51], sugeri uma solução de $1 \%$ fenolftaleína in $70 \%$ álcool etanol para determinação da profundidade carbonatada, sendo essa a dotada no presente trabalho. A fenolftaleína pode ser considerado o método mais comum usado para 
detectar a carbonatação $[24,25,47,51]$. A fenolftaleína muda de coloração em uma faixa entre 8.3 e 10.0 [24, 25, 47, 51] e tem como fórmula química: $\mathrm{C}_{20} \mathrm{H}_{14} \mathrm{O}_{4}$ [56].

Outra possibilidade de medição, é o TGA, sendo esse um método quantitativo [21, 24, 25, 57-60]. A técnica é frequentemente usada para a análise da composição de materiais, sendo que a mesma mede a perda de peso resultante da decomposição térmica de um material [21, 24, 25, 57-60].

Por exemplo, para o hidróxido de cálcio Eq.

(4), a evaporação de água (desidroxilação), e para o $\mathrm{CaCO}_{3}$, a perda de $\mathrm{CO}_{2}$ (decarboxilação) Eq. (5)[15, 21, 24].

$$
\begin{aligned}
& \mathrm{Ca}(\mathrm{OH})_{2}+\text { calor } \rightarrow \mathrm{CaO}+\mathrm{H}_{2} \mathrm{O} \\
& \mathrm{CaCO}+\text { calor } \rightarrow \mathrm{CaO}+\mathrm{CO}_{2}
\end{aligned}
$$

A perda de massa medida devido à desidroxilação pode ser adotada para o cálculo do peso de $\mathrm{Ca}(\mathrm{OH})_{2}$ presente na argamassa, considerando-se os pesos moleculares dos compostos envolvidos [15, 21]. Da mesma forma, procede-se para à decarboxilação, em que a partir da massa perdida durante o teste, podese calcular o peso de $\mathrm{CaCO}_{3}$ originalmente presente [1, 11, 15, 21]. Cada miligrama de peso perdida a partir da decomposição térmica significa uma presença de $2.272 \mathrm{mg} \mathrm{de} \mathrm{CaCO}_{3}$ [21, 61]. Para além do $\mathrm{Ca}(\mathrm{OH})_{2} \mathrm{e}$ $\mathrm{CaCO} 3$, outros compostos podem estar presentes na argamassa, maiores detalhes podem ser obtidos em OLIVEIRA [1] e LAWRENCE [24].

Uma vez que a campanha experimental destina-se a apoiar a análise multi-física, suportando o modelo desenvolvido por FERRETTI e BAŽANT [14] e aprimorado por OLIVEIRA [1], a fórmula adotada para se calcular o grau de carbonatação, considera a definição de R apresentada por aqueles autores [14]:

$$
R=\frac{\text { Mass }_{\mathrm{CaCO}_{3}}}{\text { Mass }_{\mathrm{CaCO}_{3 \text { Max }}}}
$$

Em que: $R$ é o grau de reação, $\mathrm{Massa}_{\mathrm{CaCO} 3}$ é a massa de carbonato de cálcio; e $M a s s a_{\mathrm{CaCO} m a ́ x}$ é a massa máxima de carbonato de cálcio na argamassa

Em termos gerais, o estudo das reações químicas é usualmente feito em termos de moles [62], a definição apresentada por FERRETTI e BAŽANT [14] e os resultados de TGA são expressos em termos de massa, logo a formulação adoptada foi também expressa em termos de massa, como também realizado em ARIZZI e CUTRONE [4].

Nesse sentido, no presente trabalho tanto o numerador quanto o denominador da definição de $R$ foram atualizados para cada análise, essa consideração foi adotada para uma definição mais precisa, algo que pode ser tido como um fator diferenciador nessa linha de estudos [1, 11]. Este método foi adotado uma vez que a cal, apresentou carbonato de cálcio na composição inicial (mais detalhes serão informados em sequência) e para miminizar possíveis erros experimentais [1]. Outras formulações que consideraram apenas a faixa de desidroxilação durante o teste $[4,24,63]$ não conseguem capturar por completo esse efeito.

Em termos estequimétricos, um mol de $\mathrm{Ca}(\mathrm{OH})_{2}$, quando completamente carbonatado, produz um mol de $\mathrm{CaCO}_{3}[64,65]$.

Logo, o termo Massa $a_{C a C O 3 m a ́ x}$ pode ser calculado, convertendo-se a massa de $\mathrm{Ca}(\mathrm{OH})_{2}$ para $\mathrm{CaCO}_{3}$ pela consideração das respectivas massas moleculares [66], e a equação para $R$ pode ser reescrita como [15, 67]:

$$
R=\frac{\text { Massa }_{\mathrm{CaCO}_{3}}}{\text { Massa }_{\mathrm{CaCO}_{3}}+\left(\text { Massa }_{\mathrm{Ca}(\mathrm{OH}) 2}\right) \times\left(\frac{\text { Massamolar }_{\mathrm{CaCO} 3}}{\text { Massamolar }_{\mathrm{Ca}(\mathrm{OH}) 2}}\right)}
$$

Em que: $\mathrm{Massa}_{\mathrm{Ca}(\mathrm{OH}) 2}$ é a massa de carbonato de cálcio, $\mathrm{MassaMolar}_{\mathrm{CaCO} 3}$ é a massa molecular de carbonato $\mathrm{CaCO}_{3} \approx 100 \mathrm{~g} / \mathrm{mol}$, e Massa Molar ${ }_{\mathrm{Ca}(\mathrm{OH}) 2}$ é a massa molecular de $\mathrm{Ca}(\mathrm{OH})_{2} \approx 74 \mathrm{~g} / \mathrm{mol}[65,68]$. 


\section{MATERIAIS E MÉTODOS}

\section{Componentes da argamassa e a caracterização}

Nessa seção apresenta-se informação sobre a caracterização dos componentes (cal e areia) utilizados para a preparação da argamassa, iniciando-se a descrição pela cal adotada.

Foi utilizada uma cal do tipo micronizada, fornecida pela Lusical ${ }^{\circledR}$ (Companhia Lusitana de Cal, S.A.) e classificada como CL90 Q de acordo com a norma europeia EN 459-1 [69]. A cal foi caracterizada utilizando-se a fluorescência de raios X (XRF) com o equipamento Philips ${ }^{\circledR}$ X'Unique I, e adicionalmente, caracterizada utilizando-se as análises térmicas simultâneas TGA/DTG (modelo 2960 SDT V3.0F, da TA INSTRUMENTS) [70].

Mais informações sobre a XRF podem ser encontradas em JENKINS [71] e LANGHOFF et al. [72]. No ensaio TGA/DTG, as amostras foram submetidas a uma taxa de aquecimento constante de $10^{\circ} \mathrm{C} /$ minuto desde a temperatura ambiente (cerca de $30^{\circ} \mathrm{C}$ ) até a temperatura máxima de $1100{ }^{\circ} \mathrm{C}$ ), mais informações podem ser obtidas em OLIVEIRA [1].

Considerando-se essas informações, foi possível, dessa forma determinar as faixas de temperatura correspondentes aos fenômenos de desidroxilação $\left(300-550^{\circ} \mathrm{C}\right)$ e de descarboxilação $\left(650-950{ }^{\circ} \mathrm{C}\right)$, necessárias para se obter os teores de hidróxido e carbonato de cálcio, respetivamente $[11,15,21,73]$.

No estudo da cal, os resultados da análise termogravimétrica, exibidos na Figura 2, indicam um percentual inicial de $\mathrm{Ca}(\mathrm{OH})_{2}$ e $\mathrm{CaCO}_{3}$ respetivamente de $7.5 \%$ e $11.8 \%$.

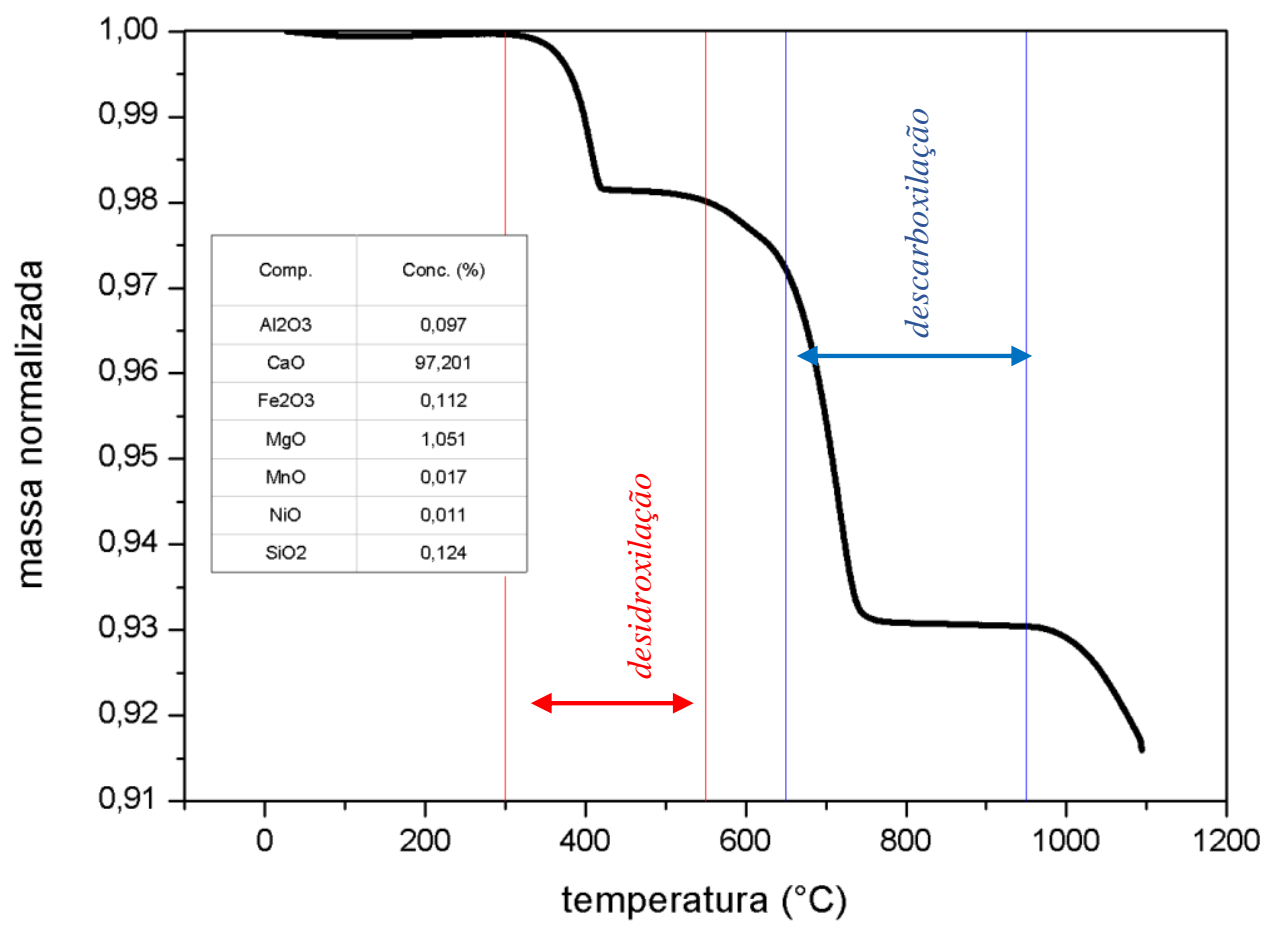

Figura 2: Caracterização termogravimétrica e química da cal aérea

Trata-se de uma percentagem significativa de $\mathrm{CaCO}_{3}$, indicando que a cal já se encontrava parcialmente carbonatada previamente à utilização na fabricação da argamassa. Os resultados obtidos por XRF, também apresentados na , indicaram a presença de $97.2 \%$ em massa de $\mathrm{CaO}$ (após normalização de resultados para $100 \%$ e não considerando os elementos de número atómico inferior a 11), e menor percentagem de $\mathrm{SO}_{3}(1.32 \%)$ e $\mathrm{MgO}(1.05 \%)$, o que confirma a predominância de compostos baseados em cálcio.

Para a areia foi adotada uma granulometria compatível com os limites referidos na norma EN-13139 [74] para aplicação em argamassas, sendo o diâmetro mínimo de $0.063 \mathrm{~mm}$ e o máximo de $1 \mathrm{~mm}$, mais in- 
formações podem ser obtidas em OLIVEIRA [1]. A composição química das areias utilizadas foi também estudada por XRF e TGA. As análises de XRF demonstraram que as areias utilizadas eram compostas predominantemente por sílica $\left(\mathrm{SiO}_{2}\right)$, em percentagem de aproximadamente $90 \%$ em massa. As análises de TGA das areias revelaram perdas de massa pequenas, consideradas insignificantes $(<0.5 \%)$, em coerência com a composição predominantemente à base de sílica reportada nos ensaios XRF. A temperatura de decomposição para sílica é de aproximadamente $200{ }^{\circ} \mathrm{C}$ [75], encontrando-se nitidamente acima do limite máximo do equipamento TGA/DTG utilizado $\left(1100^{\circ} \mathrm{C}\right)$ e permanecendo portanto indetectável [15, 21]. Nesse sentido, pode-se assumir que, as perdas de massa durante o ensaio de TGA da argamassa serão devido aos processos químicos referentes à cal, mais detalhes sobre a caracterização do material podem ser obtidos em OLIVEIRA [1].

\section{Composição da argamassa e processo de mistura}

Apresenta-se nessa seção informação sobre a composição e produção da argamassa adotados nesse trabalho. Foi escolhida uma argamassa com a composição 1:1.3:3 (cal:água:agregado) em termos de volume. Esta escolha resultou de um processo iterativo com três tentativas prévias (ver Tabela 1), tendo em conta considerações relativas a aspetos tão variados como a trabalhabilidade, a ocorrência de fissuras, bem com o respeito pelas proporções de mistura habitualmente encontradas em construções históricas [21, 36], uma vez que acordo com diferentes [1,12,24] autores a proporção 1:3 (cal:agregado) é a mais comum em pesquisas bem como representa adequadamente composições utilizados no passado, ou seja nas argamassas históricas.

Tabela 1: Composição das figuras misturas

\begin{tabular}{l|l|l}
\hline $\begin{array}{l}\text { COMPOSIÇÃO (VOLUME) } \\
\text { CAL:ÁGUA:AGREGADO }\end{array}$ & VALOR & NOTA \\
\hline Mistura 1 - 1:1:3 & teste descartado & mistura seca com baixa trabalhabilidade \\
\hline Mistura 2 - 1:1.5:3 & $173 \mathrm{~mm}$ & baixa consistência pela falta de água \\
\hline Mistura 3 - 1:1.4:3 & $135 \mathrm{~mm}$ & fissuras para corpos-de-prova com dimensões maiores \\
\hline Mistura 4 - 1:1.3:3 & $122 \mathrm{~mm}$ & adequado \\
\hline
\end{tabular}

Para todas as composições as massas específicas aparentes apresentaram valores $1800-2000 \mathrm{~kg} / \mathrm{m}^{3}$, de acordo com a EN 1015-2 [76] os valores expectáveis são da ordem de $175 \pm 10 \mathrm{~mm}$. Nesse caso, apenas a composição com maior teor de água adequava-se ao requerimento. Ainda que a mistura com 1:1.3:3 não esteja adequada a trabalhabilidade, essa foi adotada como a mistura final, para se prevenir a formação de fissuras. Valores similares de massas específicas aparentes para argamassa fresca foram obtidos por SEABRA et al. [77] e ALGARVIO [78].

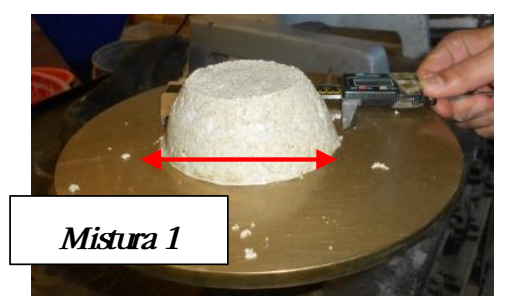

(a)

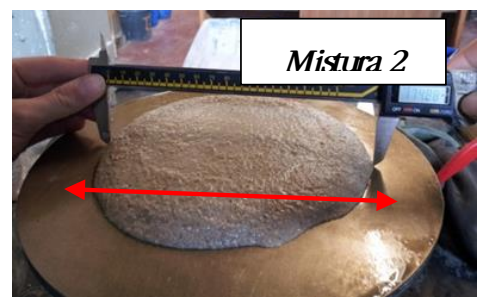

(b)

Figura 3: Ensaio flow table (a) Mistura 1 (b) Mistura 2

Mais detalhes sobre a definição da composição e do preparo da argamassa, podem ser consultados em OLIVEIRA [1]. O método de mistura adotado no presente trabalho foi o "hot-lime mix", em coerência com vários trabalhos de investigação anteriores [79-84] e também com a estratégia utilizada em construções históricas [79, 81, 82]. O processo de mistura foi feito de acordo com as recomendações da norma EN 196-1 [85], e seguiu a adição dos componentes na seguinte ordem: agregados (mistura de areias grossa e fina), cal e água, mais detalhes podem ser vistos em OLIVEIRA [1]. As misturas foram realizadas numa misturadora Würk ${ }^{\circledR}$ 
com capacidade de 3 litros e pá de mistura vertical, sendo o tempo total de amassadura de 300 segundos, mais detalhes podem ser vistos em OLIVEIRA [1].

Quanto ao problema de retração enfrentado em alguns corpos-de-prova, para esse tipo de argamassa, a dessecação (perda de água) é a principal causa [86], nesse sentido a adoção de corpos-de-prova com maiores dimensão não foi realizada, para mais detalhes ver OLIVEIRA [1].

A composição escolhida foi inicialmente preparada em termos de volume. Para uma composição mais preciso, a composição equivalente das matérias-primas em termos de peso foi finalmente realizada. Para este fim, a quantidade de materiais em termos de volume foi ponderada e a proporção equivalente expressa em função do peso foi encontrada: 1: 1.53: 6.14. Os materiais apresentaram os seguintes valores para a massa específica aparente (massa dividida pelo volume total): cal $\approx 0.85 \mathrm{~g} / \mathrm{cm}^{3}$ e areia $\approx 1.74 \mathrm{~g} / \mathrm{cm}^{3}$.

\section{Influência da dimensão do corpo-de-prova sobre o processo de carbonatação}

Definida a composição da argamassa, inicia-se um programa experimental com o objetivo de avaliar a influência do tamanho do corpo-de-prova na evolução da frente de carbonatação. Para isso foram preparados vários corpos-de-prova cilíndricos de argamassa com a proporção diâmetro/altura de 1:2, e diâmetro variável entre $3.6 \mathrm{~cm}$ (corpo de prova S.A) e $15 \mathrm{~cm}$ (corpo de prova S.E).

Todos os corpos-de-prova foram moldados com massa específica aparente inicial igual a $2000 \mathrm{~kg} / \mathrm{m}^{3}$; uma vez que a compactação do material poderia influenciar na estrutura da estrutura do material. A compactação com realizada com 10 golpes por camada de material $(\sim 2 \mathrm{~cm})$. O processo foi feito com a ajuda de um dispositivo metálico com um peso de $\sim 250 \mathrm{~g}$ e uma área de contato de $\sim 12,56 \mathrm{~cm}^{2}$, ou com um dispositivo auxiliar com o mesmo peso e uma área de contato de $0,79 \mathrm{~cm}^{2}$.

Os corpos-de-prova (CP's) foram mantidos em moldes de polipropileno e selados com filme plástico, permanecendo isolados das condições atmosféricas durante 3 dias, esse foi o tempo necessário para que os corpos de prova obtivessem a adequada resistência a retirada da forma. Em seguida, foram expostos ao ambiente laboratorial (câmara climática, com temperatura $=20^{\circ} \mathrm{C}$, umidade $=60 \%$ e concentração ambiente de $\left.\mathrm{CO}_{2}\right)$, por forma a possibilitar os processos de secagem e carbonatação. Para cada idade de ensaio $(10,21 \mathrm{e}$ 90 dias) foi quebrado um exemplar de cada diâmetro para o ensaio com o indicador fenolftaleína, sendo a profundidade carbonatada medida com paquímetro digital (mais detalhes podem ser visualizados em Oliveira [1]).

\section{Corpos-de-prova com espessura reduzida}

O processo de difusão da umidade e de carbonatação tanto para materiais cimentícios quanto para argamassas à base de cal estão associados $[14,21,28,87-90]$. Com o intuito de se reduzir a possível influência da umidade no processo de carbonatação, discos com $3.8 \mathrm{~cm}$ de diâmetro e espessura de $0.8 \mathrm{~cm}$ foram fabricados, detalhes são mostrados na Figura 4.

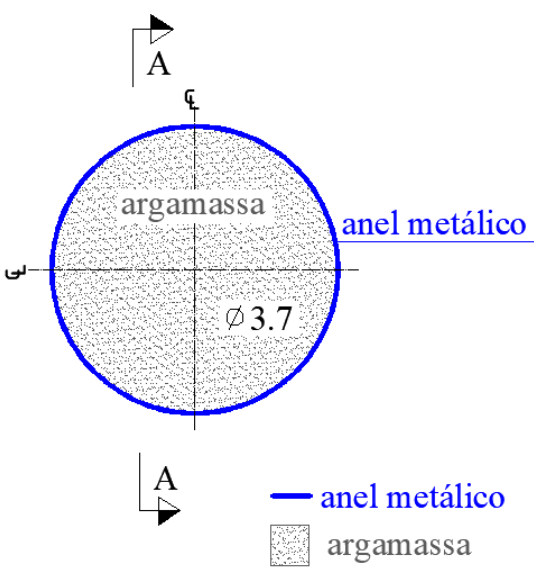

(a)

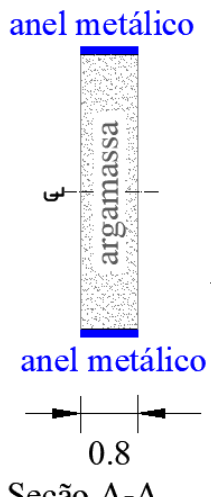

Seção A-A

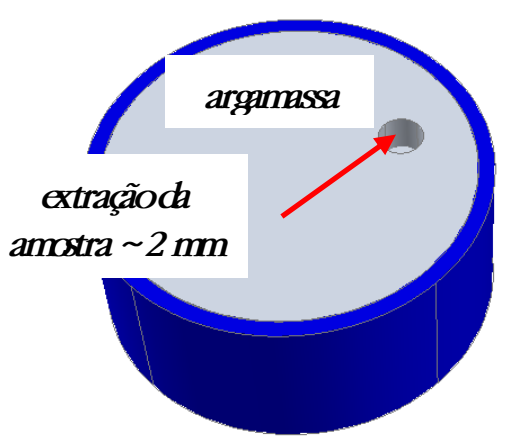

(b)

Figura 4: Disco para estudo da carbonatação (dimensões em $\mathrm{cm}$ ) - a) Geometria - b) Representação da retirada das amostras: profundidade $\sim 2 \mathrm{~mm}$. 
Para a avaliação da carbonatação da argamassa presente nos discos, a análise termogravimétrica foi escolhida. Para todos os testes TGA, os procedimentos foram realizados em atmosfera de argônio, com um aumento de temperatura de $10^{\circ} \mathrm{C} / \mathrm{min}$, uma vez que esse valor está em coerência com outros autores, para além disso, foram realizados ensaios que investigaram a influência desse parâmetro nos resultados. Testes foram realizados variando-se a taxa de incremento de temperatura entre $5-20^{\circ} \mathrm{C} / \mathrm{min}(5,7.5(2 \times), 10(2 \times), 15$, $20^{\circ} \mathrm{C} / \mathrm{min}$ ), e os resultados, em termos de grau de reação (para a formulação utilizada para calcular o R, ver Eq. (7)), apresentaram diferença máxima nos valores de $~ 5 \%$; mostrando que a velocidade de execução dos testes possui pouca relevância no resultado final, mais detalhes podem ser visualizados em OLIVEIRA [1].

As temperaturas máximas durante os testes TGA foram 1000 ou $1100{ }^{\circ} \mathrm{C}$, como em algumas experiências, o valor de temperatura mais baixo $\left(1000{ }^{\circ} \mathrm{C}\right)$ foi adotado por simplicidade e para testes mais rápidos. Não foi observada alteração significativa nos resultados, porque esses valores estão fora dos intervalos de processos de desidroxilação / descarboxilação. O discos foram armazenados em três ambientes distintos, com condições diferentes: padrão (umidade $=60 \%$, temperatura $=20^{\circ} \mathrm{C}$ e $\left[\mathrm{CO}_{2}\right]=0.035 \%$ ), elevada umidade (umidade $=90 \%$, temperatura $=20{ }^{\circ} \mathrm{C}$ e $\left[\mathrm{CO}_{2}\right]=0.035 \%$ ), e alta concentração de gás carbônico (umidade $=$ $60 \%$, temperatura $=20^{\circ} \mathrm{C}$ e $\left[\mathrm{CO}_{2}\right]=4 \%$ ). Os discos foram expostos aos ambientes desde a moldagem argamassa.

\section{RESULTADOS}

Nessa seção apresenta-se os principais resultados. No tocante ao ensaio com fenolftaleína nos CPs de diferentes diâmetros, os resultados apresentados na Tabela 2 indicam a existência influência do diâmetro dos cilindros sobre a profundidade carbonatada para a primeira idade testada, ou seja, aos 10 dias. Nessa idade, o cilindro da série S.A (36 mm de diâmetro) apresentou uma profundidade carbonatada aproximadamente duas vezes superior à do exemplar $(S . D)$ com $87 \mathrm{~mm}$ de diâmetro. Verificou-se também uma tendência contínua de aumento da profundidade carbonatada com a diminuição do diâmetro do corpo-de-prova para todas as medições efetuadas aos 10 dias de idade. Para os testes realizados nas idades de 21 e 90 dias, não se verificou o mesmo tipo de tendência, uma vez que as medições de profundidade carbonatada foram aproximadamente idênticas em todos os cilindros ensaiados (para cada uma das duas idades em questão).

A maior profundidade de carbonatação foi observada no corpo-de-prova de $36 \mathrm{~mm}$ de diâmetro para os 10 dias de idade (quando comparado com os exemplares de maior diâmetro), esse resultado pode ser parcialmente justificado pela secagem mais rápida deste corpo-de-prova nas fases iniciais, criando caminhos que facilitam a difusão do $\mathrm{CO}_{2}$, e portanto acelerando o processo de carbonatação interna. A secagem nas regiões superficiais nos exemplares estudados de diâmetro maior, por ser considerada mais lenta devido à migração interna de água das zonas interiores para as regiões superficiais em processo de secagem, atrasando o esvaziamento da rede porosa, e portanto limitando as condições para que ocorra a progressão da difusão do $\mathrm{CO}_{2}[1$, 67]. Este efeito retardador tende a reduzir a importância nas idades mais avançadas (21 e 90 dias) conforme observado experimentalmente. Conclusões similares entre o processo secagem e a carbonatação são apresentadas em diferentes trabalhos, tanto no campo experimental [1, 11, 27, 91, 92] quanto numérico [87-89, 93, 94].

A Figura 5 apresenta a evolução da carbonatação para os corpo-de-prova da série $S . A$ para três idades, 10; 21 e 90 dias. 
Tabela 2: Estudo do efeito do tamanho das amostras para a profundidade de carbonatação

\begin{tabular}{|c|c|c|c|c|}
\hline IDADE & $\mathrm{CP}$ & DIÂMETRO (mm) & $\begin{array}{l}\text { MASSA ESPECÍFICA } \\
\text { APARENTE }\left(\mathrm{kg} / \mathrm{m}^{3}\right)\end{array}$ & $\begin{array}{c}\text { PROFUNDIDADE } \\
\text { CARBONATADA }(\mathrm{mm})\end{array}$ \\
\hline \multirow{5}{*}{10 Dias } & S.A & 36 & 1761 & $\sim 3.9$ \\
\hline & S.B & 60 & 1820 & $\sim 2.7$ \\
\hline & S.C & 71 & 1852 & $\sim 2.4$ \\
\hline & S.D & 86 & 1852 & $\sim 1.8$ \\
\hline & S.E & 150 & \multicolumn{2}{|c|}{$\mathrm{CP}$ danificado durante o procedimento experimental } \\
\hline \multirow{5}{*}{21 Dias } & S.A & 37 & 1820 & $\sim 6.0$ \\
\hline & S.B & 60 & 1858 & $\begin{array}{l}5.9 \\
\sim\end{array}$ \\
\hline & S.C & 72 & 1856 & $\sim 5.8$ \\
\hline & S.D & 87 & 1850 & $\sim 5.9$ \\
\hline & S.E & 150 & 1860 & $\sim 6.0$ \\
\hline \multirow{5}{*}{90 Dias } & S.A & 36 & 1831 & $\sim 12.3$ \\
\hline & S.B & 60 & 1858 & $\sim 12.1$ \\
\hline & S.C & 72 & 1856 & $\sim 11.9$ \\
\hline & S.D & 87 & 1850 & $\sim 12.1$ \\
\hline & S.E & 150 & 1860 & $\sim 12.0$ \\
\hline
\end{tabular}

(A nomenclatura S.A, S.B, S.C S.D e S.E corresponde respectivamente aos 5 espécimes testados e a letra $\mathrm{S}$ corresponde ao termo em inglês "specimen”)

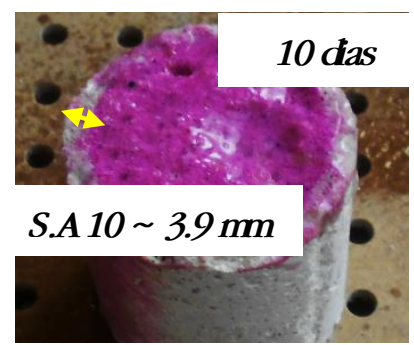

(a) 10 dias $-S . A 10$

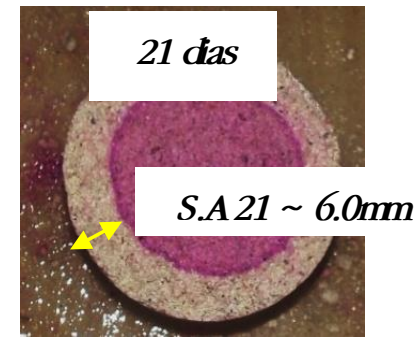

(b) 21 dias - S.A 21

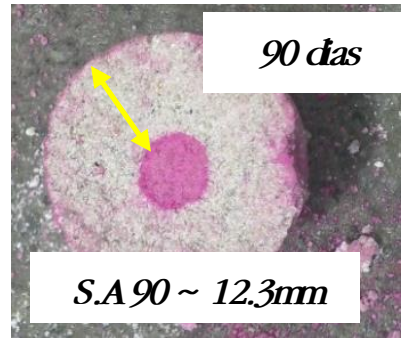

(c) 90 dias - S.A90

Figura 5: Profundidade carbonatada - Corpos-de-prova S.A: 10; 21 e 90 dias - As indicações correspondem a profundidade carbonatada.

\section{Estudo da carbonatação em discos com reduzida espessura}

A influência da amostragem na carbonatação também foi investigada, para esse estudo, três amostras coletadas o mesmo disco (corpo-de-prova) na mesma idade, aos 20 dias, e com o mesmo procedimento de amostragem. A amostragem foi feita na superfície do disco. As amostras testadas tiveram respectivamente a massa inicial de 54,20 mg (T.A), 85,69 $\mathrm{mg}(T . B)$ e $67,92 \mathrm{mg}(T . C)$. As três amostras foram testadas na faixa de $20-1100{ }^{\circ} \mathrm{C}$, e os resultados são mostrados na Figura 6 (A nomeclatura T.A, T.B e T.C correspondem respectivamente aos 3 espécimes testados, sendo a letra $T$, correspondente ao ensaio de termogravimetria). 
Figura 6: Verificação da influência da amostragem - 3 amostras (T.A, T.B eT.C)

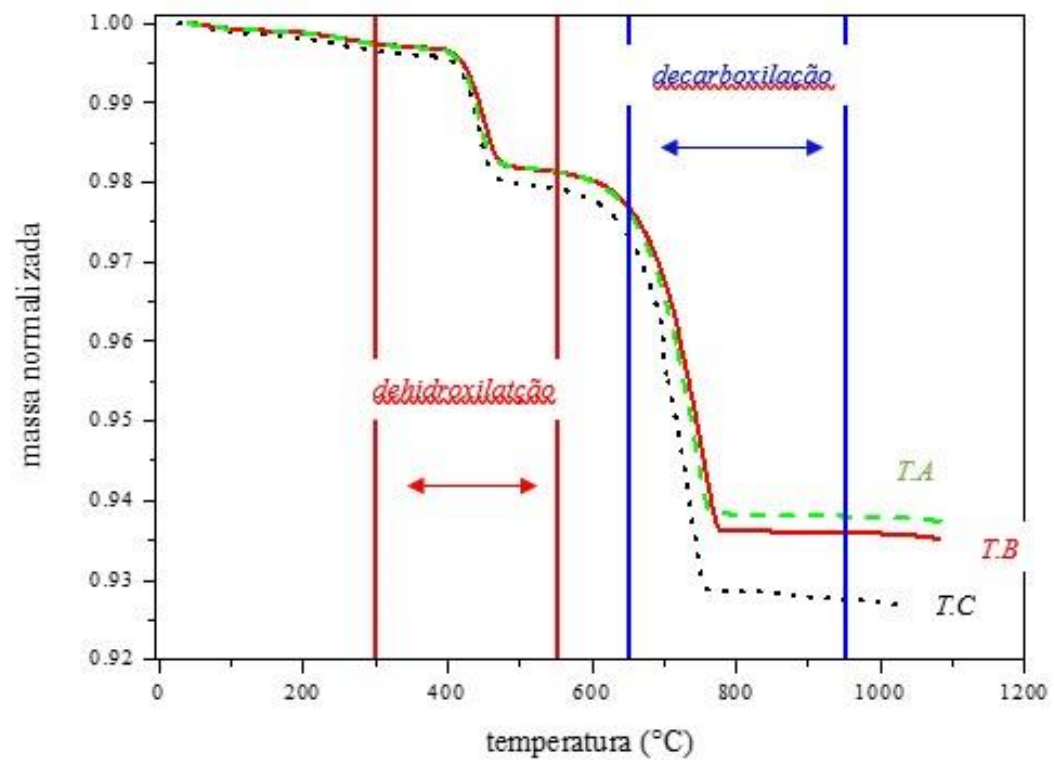

A dispersão dos resultados pode ser considerada aceitável, uma vez que apresentou valores relativamente baixos em termos de graus de reação, 3\%, sendo os valores de R, para TA, TB e TC foram respectivamente $\sim 58 \%, \sim 60 \%$ e $\sim 61 \%$. Esta informação permitiu considerar que o processo de amostragem tem pouca influência nos resultados.

Amostras para os ensaios de TGA foram colhidas dos discos em sete diferentes datas: 1, 3, 7, 14, 21 e 28 dias. As amostras foram coletadas nos primeiros 2 milímetros, com a utilização de um extrator metálico com uma marca de referência (para maiores detalhes ver OLIVEIRA [1]). O resumo das informações contento dos pesos iniciais das amostras testadas pode ser visualizado na Tabela 3.

A conversão de $\mathrm{Ca}(\mathrm{OH})_{2}$ para $\mathrm{CaCO}_{3}$ acontece lentamente [95-97] e usualmente de forma incompleta [21, 98-102], nesse sentido que concerne a carbonatação, a normalização foi realizada [1, 11]. Nesse sentido o valor de $R$ para cada teste foi dividido pela valor máximo da reação para cada tipo de grupo de testes, ou seja $R_{\text {normalizado }}=R / R_{\max }$; em que $R_{\max }$ é o valor máximo de $R$ para cada grupo de experimento. Esse processo de normalização foi também utilizado por Oliveira [1].

Tabela 3: Amostras coletadas para análise termogravimétrica

\begin{tabular}{c|c|c|c}
\hline \multirow{2}{*}{$\begin{array}{c}\text { IDADE } \\
\text { (DIAS) }\end{array}$} & $\begin{array}{c}\mid c \\
\text { AMBIENTE } \\
\text { PADRÃO }\end{array}$ & $\begin{array}{c}\text { AMBIENTE ÚMIDO } \\
(\mathbf{H}=\mathbf{9 0} \%)\end{array}$ & $\begin{array}{c}\text { ALTA CONCENTRAÇÃO } \\
\text { DE CO }_{2}\end{array}$ \\
\hline 1 & 62.82 & 55.67 & 57.52 \\
\hline 4 & 58.66 & 59.78 & 57.56 \\
\hline 7 & 73.85 & 70.42 & 62.65 \\
\hline 14 & 61.39 & 80.02 & 58.28 \\
\hline 21 & 69.18 & 68.37 & 77.24 \\
\hline 28 & 71.94 & 56.44 & 67.55 \\
\hline
\end{tabular}


Os resultados após a normalização, para cada um dos três ambientes estudados são mostrados na Figura 7 .

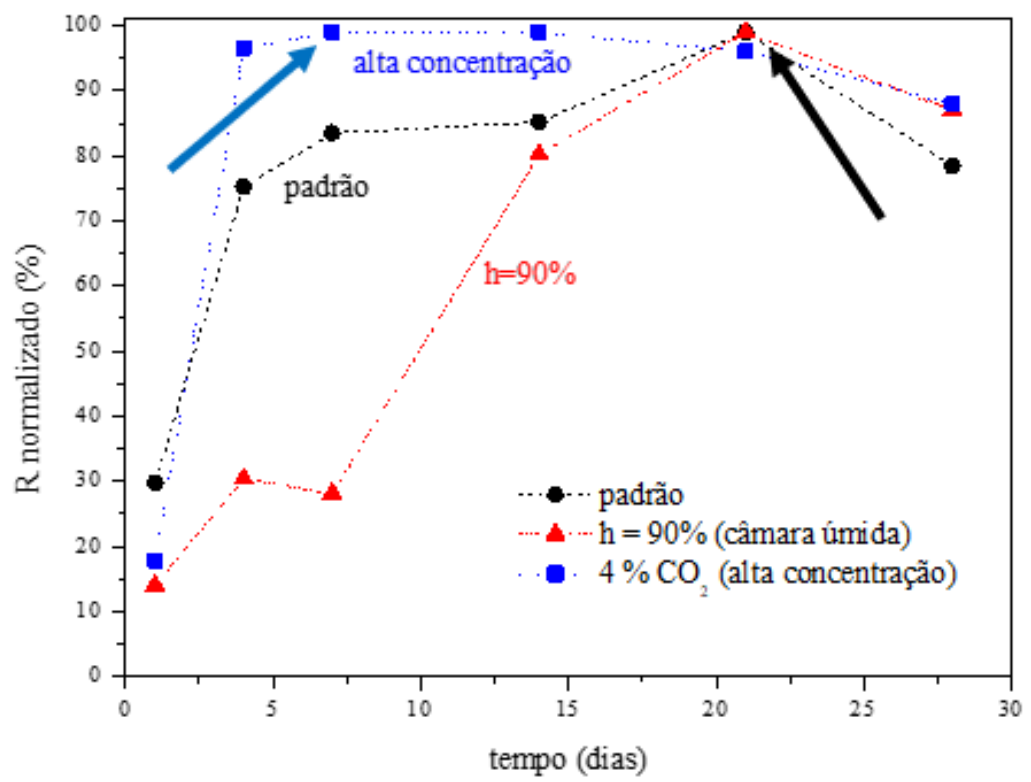

Figura 7: Perfil de carbonatação em discos - três ambientes de estudo (padrão, câmara úmida e alta concentração de $\mathrm{CO}_{2}$ ) - Valores máximos após a normalização para cada ambiente estão indicados por setas

Os resultados em termos de massa de carbonato, e dióxido de cálcio juntamente com o percentual de carbonatação antes e depois da normalização estão mostrados na Tabela 4. Para o percentual de $\mathrm{CaCO}_{3}$, as Equações 6 e 7 foram utilizadas, maiores detalhes podem ser visualizados em OLIVEIRA et al. [11].

Durante a medição do grau de reação, as amostras apresentaram distintos valores máximo para o grau de reação, $\mathrm{R} \sim 76 \%$ e $\mathrm{R} \sim 69 \%$, respetivamente para as câmara padrão e de elevado $\mathrm{CO}_{2}$, em contrapartida para o ambiente com elevada umidade foi obtido um $R \sim 99 \%$ (ver Tabela 4 ). A formação de uma camada protetora de carbonato, pode explicar a reação incompleta, em diferentes estudos [100, 103, 104], cita-se que usualmente a percentagem de conversão é menor que $80 \%$ em condições usuais [100, 103, 104].

Os discos armazenados na câmara úmida apresentaram um início de reação mais lento, aproximadamente até o sétimo dia, em comparação com os discos armazenados nas outras condições, em sequência houve um aumento progressivo até o teste realizado após 14 dias.

Esse resultado pode ser explicado pelo fato que a elevada umidade nos poros bloqueia a entrada de $\mathrm{CO}_{2}$, que é essencial para o progresso da carbonatação. Em contrapartida, os discos armazenados nas câmaras padrão e com elevada concentração de $\mathrm{CO}_{2}$ tiveram um comportamento similar, mas tendo o disco em ambiente de elevado $\mathrm{CO}_{2}$ um progresso mais rápido e com maior percentual de carbonatação.

Durante a realização dos experimentos, para os três ambientes estudados, houve um decréscimo não esperado para o grau de reação para as medições realizadas a 28 dias em comparação com as medições feitas na data de 21 dias.

Tabela 4: Resultados análise termogravimétrica - Teor de $\mathrm{Ca}(\mathrm{OH})_{2}, \mathrm{CaCO}_{3}$, e percentuais de carbonatação 
antes de depois do processo de normalização.

\begin{tabular}{|c|c|c|c|c|c|}
\hline \multicolumn{6}{|c|}{ AMBIENTE PADRÃO } \\
\hline \multirow{7}{*}{ 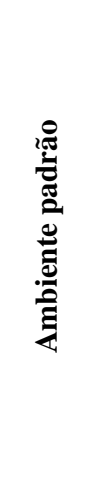 } & IDADE & $\begin{array}{c}\text { MASSA CA }(\mathrm{OH})_{2} \\
\text { (MG) }\end{array}$ & $\begin{array}{l}\text { MASSA CACO }_{3} \\
\text { (MG) }\end{array}$ & $\begin{array}{c}\text { PERCENTUAL CARBO- } \\
\text { NATADO (\%) }\end{array}$ & $\begin{array}{c}\text { PERCENTUAL CARBO- } \\
\text { NATADO NORMALIZA- } \\
\text { DO (\%) }\end{array}$ \\
\hline & 1 & 5.86 & 1.87 & 19.12 & 24.99 \\
\hline & 4 & 5.15 & 8.15 & 53.95 & 70.53 \\
\hline & 7 & 4.51 & 9.59 & 61.14 & 79.93 \\
\hline & 14 & 4.58 & 10.4 & 62.68 & 81.94 \\
\hline & 21 & 2.36 & 10.38 & 76.49 & 100.00 \\
\hline & 28 & 5.16 & 9.14 & 56.72 & 74.16 \\
\hline \multicolumn{6}{|c|}{ AMBIENTE ÚMIDO (H=90\%) } \\
\hline \multirow{7}{*}{ 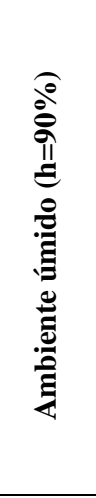 } & IDADE & $\begin{array}{c}\text { MASSA CA }(\mathrm{OH})_{2} \\
\text { (MG) }\end{array}$ & $\begin{array}{c}\text { MASSA CACO }_{3} \\
\text { (MG) }\end{array}$ & $\begin{array}{c}\text { PERCENTUAL CARBO- } \\
\text { NATADO (\%) }\end{array}$ & $\begin{array}{c}\text { PERCENTUAL CARBO- } \\
\text { NATADO NORMALIZA- } \\
\text { DO (\%) }\end{array}$ \\
\hline & 1 & 7 & 1.14 & 10.72 & 10.76 \\
\hline & 4 & 6.55 & 2.85 & 24.35 & 24.43 \\
\hline & 7 & 8.05 & 3.13 & 22.33 & 22.40 \\
\hline & 14 & 2.81 & 11.22 & 74.71 & 74.97 \\
\hline & 21 & 0.04 & 16.12 & 99.65 & 100.00 \\
\hline & 28 & 1.77 & 11.59 & 82.93 & 83.22 \\
\hline \multicolumn{6}{|c|}{ AMBIENTE COM ALTA CONCENTRAÇÃO DE $\mathrm{CO}_{2}$} \\
\hline \multirow{7}{*}{ 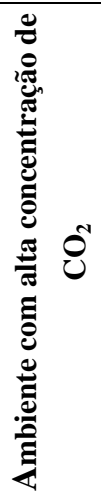 } & IDADE & $\begin{array}{c}\text { MASSA CA }(\mathrm{OH})_{2} \\
\text { (MG) }\end{array}$ & $\begin{array}{c}\text { MASSA CACO }_{3} \\
\text { (MG) }\end{array}$ & $\begin{array}{c}\text { PERCENTUAL CARBO- } \\
\text { NATADO (\%) }\end{array}$ & $\begin{array}{c}\text { PERCENTUAL CARBO- } \\
\text { NATADO NORMALIZA- } \\
\text { DO (\%) }\end{array}$ \\
\hline & 1 & 8.67 & 1.33 & 10.22 & 14.83 \\
\hline & 4 & 3.72 & 9.77 & 66.01 & 95.80 \\
\hline & 7 & 4.04 & 11.97 & 68.65 & 99.62 \\
\hline & 14 & 3.14 & 9.40 & 68.91 & 100.00 \\
\hline & 21 & 3.52 & 9.04 & 65.52 & 95.09 \\
\hline & 28 & 5.21 & 10.1 & 58.94 & 85.54 \\
\hline
\end{tabular}

\section{DISCUSSÃO}

Os resultados obtidos em ambos os experimentos indicam a dependência da evolução do processo de carbonatação das condições ambientais e do interior do material. Os dados mostram a conexão direta entre a umidade e a carbonatação.

No estudo do efeito do tamanho do $C P$, a influência da umidade concentra-se nas idades iniciais, visto que a difusão da umidade ainda está elevada e o processo está na fase inicial [1, 11, 67], apresentando um fluxo da parte interior para a exterior.

A reação de carbonatação desenvolve-se mais rapidamente em condições com maior presença de água [33, 40, 105, 106]. Por outro lado, em condições totalmente saturadas, a reação torna-se mais lenta [27]. No presente estudo, para os discos de pequena espessura a elevada presença de água também parece bloquear a inicialização da carbonatação, mas após o processo se iniciar, o percentual de carbonatação é quase completo $(\sim 99 \%)$ para esse ambiente.

Estudos na literatura indicam que numa atmosfera com aproximadamente $100 \%$ de concentração $\mathrm{CO}_{2}$, 
a carbonatação ocorre na superfície da cal de forma muito rápida, estagnando em seguida, devido à rápida produção de precipitado na superfície, o que impede a entrada de mais $\mathrm{CO}_{2}$ [27]. Os resultados obtidos na presente pesquisa mostram coerência com os resultados da literatura.

\section{CONCLUSÕES}

A cal aérea é um material que vem sendo utilizado a séculos em diferentes regiões. O comportamento físico e mecânico das argamassas fabricadas com esse material, dependem diretamente do processo de carbonatação. Uma vez que a maior parcela das deformações das alvenarias antigas ocorre nas argamassas, o referido processo tem torna-se de considerável relevância para o estudo do comportamento estrutural, para além disso, quando se pretende utilizar o material no restauro, torna-se fundamental o conhecimento dessa evolução. Diferentes estudos mostram que há significativo espaço para a utilização da cal aérea, tanto para a recuperação de estruturas históricas, ou em aplicações em construções recentes, diferentes estudos indicam essa aplicabilidade.

No presente estudo, a carbonatação de argamassas de cal aérea foi estudada por duas técnicas diferentes, a fenolftaleína e a análise termogravimétrica.

- Os cilindros com cinco diferentes diâmetros apresentaram resultados diferenciados para o primeiro instante de medição (aos 10 dias de idade), com os corpos-de-prova de menor diâmetro a exibirem maiores profundidades de carbonatação. Contudo, para idades mais avançadas essa dependência diminuiu, tendo-se obtido profundidades de carbonatação muito próximas para todas as dimensões de corpos-de-prova aos 21 e 90 dias de idade. A carbonatação mais acelerada dos corpos-de-prova menores poderá estar relacionada com a secagem mais rápida, nesse sentido, propiciando condições melhoradas para a carbonatação.

- Os discos armazenados na câmara com elevada umidade apresentaram um retardamento inicial no processo de carbonatação, mas em contrapartida um grau de conversão mais elevado quando comparado com os discos armazenados nas câmaras padrão e com elevada concentração de $\mathrm{CO}_{2}$.

- Em termos de grau de reação, os discos armazenados na câmara úmida apresentaram um $R \sim 99 \%$, enquanto que para os demais ambientes, antes da normalização, um grau de reação de $R \sim 76 \%$ e $R \sim 69 \%$, respetivamente para o ambiente padrão e com elevada concentração de $\mathrm{CO}_{2}$. A reação incompleta, para condições padrão já havia sido observada na literatura por diferentes autores e foi mais uma vez observada no presente trabalho.

- Comportamentos distintos foram oberados na literatura, quando amostras foram armazenadas em ambientes com concentração ambiente de $\mathrm{CO}_{2}$ e com elevado teor desse gás. A interdependência da carbonatação e umidade fica evidente em ambos os ensaios.

Apesar do número de pesquisas relacionadas a cal estar crescendo, esse ainda é bastante reduzido quando comparado com estudos relacionados ao concreto. Inúmeros trabalhos apontam a aplicabilidade da cal desse material. Esse é um tema que vêm sendo estudado na Universidade do Minho e que inicia-se na Universidade Federal de Minas Gerais.

\section{AGRADECIMENTOS}

Os autores agradecem o financiamento fornecido pelo programa "Ciência sem Fronteiras" apoiado pelo Conselho Nacional de Desenvolvimento Científico e Tecnológico (CNPq), bem como a FCT, Fundação para Ciência e Tecnologia.

\section{BIBLIOGRAFIA}

[1] OLIVEIRA, M.A., A Multi-Physics Approach Applied to Masonry Structures with Non-Hydraulic Lime Mortars, in Departamento de Engenharia Civil, Universidade do Minho: Guimarães, Portugal, 2016.

[2] WARREN, M., Economic Analysis for Property and Business. First Edition ed: Butterworth-Heinemann, Routledge, 2000.

[3] ARIZZI, A., CULTRONE, G. "The influence of aggregate texture, morphology and grading on the carbonation of non-hydraulic (aerial) lime-based mortars". Quarterly Journal of Engineering Geology and Hydrogeology. v. 46, n. 4, pp. 507-520, 2013.

[4] ARIZZI, A. , CULTRONE, G. "The difference in behaviour between calcitic and dolomitic lime mortars set under dry conditions: The relationship between textural and physical-mechanical properties". Cement and Concrete Research. v. 42, n. 6, pp. 818-826, 2012. 
[5] CEN, CEN-EN 459-1, in Part 1: Definitions, specifications and conformity criteria, European Committee for Standardization, 2010.

[6] ASTM, C51-11 - Standard Terminology Relating to Lime and Limestone (as used by the Industry), in Subcommittee: C07.08, American Society for Testing and Materials: ASTM International 2011.

[7] FARIA, P., HENRIQUES, F., RATO, V. "Comparative evaluation of aerial lime mortars for architectural conservation". Journal of Cultural Heritage. v. 9, n. 3, pp. 338-346, 2008.

[8] EL-REEDY, M., Steel-Reinforced Concrete Structures: Assessment and Repair of Corrosion: CRC Press, 2007.

[9] LANAS, J., ALVAREZ, J.I. "Masonry repair lime-based mortars: factors affecting the mechanical behavior". Cement and Concrete Research. v. 33, n. 11, pp. 1867-1876, 2003.

[10] MOROPOUlOU, A., BAKOLAS, A., MOUNDOUlAS, P., et al., "Strength development and lime reaction in mortars for repairing historic masonries". Cement and Concrete Composites. v. 27, n. 2, pp. 289294, 2005.

[11] OLIVEIRA, M.A., AZENHA, M., LOURENÇO, P.B., et al., "Experimental analysis of the carbonation and humidity diffusion processes in aerial lime mortar". Construction and Building Materials. v. 148, pp. 3848, 2017.

[12] OLIVEIRA, M.A., AZENHA, M., LOURENÇO, P.B., et al., Study of the evolution of carbonation in aerial lime mortars in V Fical - FICAL - FORUM IBERICO DA CAL. Lisbon, 2016.

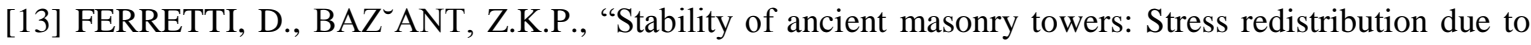
drying, carbonation, and creep". Cement and Concrete Research. v. 36, n. 7, pp. 1389-1398, 2006.

[14] FERRETTI, D., BAŽANT, Z.P. "Stability of ancient masonry towers: Moisture diffusion, carbonation and size effect". Cement and Concrete Research. v. 36, n. 7, pp. 1379-1388, 2006.

[15] OLIVEIRA, M.A., AZENHA, M., LOURENÇO, P.B., et al., Study of the evolution of carbonation in aerial lime mortars (in portuguese), in V Fical - FICAL - FORUM IBÉRICO DA CAL: Lisbon, 2016.

[16] COWPER, A.D., Lime and lime mortars. Reprint of 1927 edition, Illustrated edition ed, Donhead: Shaftsbury: Donhead Publishing Ltd, 1998.

[17] PAIVA, H., MARQUES, E., VELOSA, A., et al., Preliminary studies on the development of lime based mortars for adobe masonry, Heritage, in Weathering and Conservation, M.A.d.B. Rafael Fort, Miguel Gomez-Heras, Carmen Vazquez-Calvo, Editor: Madrid, Spain, 2006.

[18] SILVA, A.S., RICARDO, J.M., SALTA, M., et al., Characterization of Roman mortars from the historical town of Mértola, in Heritage Weathering and Conservation, V.-C. Alvarez de Buergo; GomesHeras, Editor. 2006, Taylor \& Francis: Madrid, Spain. p. 85-90

[19] PACHECO TORGAL, F., MIRALDO, S., LABRINCHA, J.A., et al., "An overview on concrete carbonation in the context of eco-efficient construction: Evaluation, use of SCMs and/or RAC". Construction and Building Materials. v. 36, pp. 141-150, 2012.

[20] COWPER, A.D., Lime and lime mortars. Reprint of 1927 edn: Shaftsbury: Donhead Publishing Ltd, 1998.

[21] OLIVEIRA, M.A., A Multi-Physics Approach Applied to Masonry Structures with Non-Hydraulic Lime Mortars, in Civil Enginnering, University of Minho: Guimarães, Portugal, 2015.

[22] GARAVAGliA, E., ANZANI, A., BINDA, L. "Probabilistic Model for the Assessment of Historic Buildings under Permanent Loading”. Journal of Materials in Civil Engineering. v. 18, n. 6, pp. 858-867, 2006.

[23] ANZANI, A., GARAVAGLIA, E., BINDA, L. "Long-term damage of historic masonry: A probabilistic model". Construction and Building Materials. v. 23, n. 2, pp. 713-724, 2009.

[24] LAWRENCE, R.M.H., A Study of Carbonation in Non-Hydraulic Lime Mortars, in Department of Architecture and Civil Engineering, University of Bath: Bath, 2006.

[25] LAWRENCE, R.M.H., MAYS, T.J., WALKER, P., et al., "Determination of carbonation profiles in non-hydraulic lime mortars using thermogravimetric analysis". Thermochimica Acta. v. 444, n. 2, pp. 179189,2006

[26] ANDREJKOVICOVA, S., FERRAZ, E., VELOSA, A.L., et al., "Air Lime Mortars with Incorporation of Sepiolite and Synthetic Zeolite Pellets". v. 9, n. 1, pp. 79-91, 2012.

[27] MOOREHEAD, D.R., "Cementation by the carbonation of hydrated lime". Cement and Concrete Research. v. 16, n. 5, pp. 700-708, 1986.

[28] VAN BALEN, K., VAN GEMERT, D., "Modelling lime mortar carbonation". Materials and Structures. v. 27, n. 27, pp. 393-398, 1994.

[29] VAN GERVEN, T., CIZER, O., MERTENS, G., et al., Mineral Carbonation at K.U.Leuven, in $1^{\text {st }}$ International Slag Valorisation Symposium: Leuven, Belgium, 6-7/4/2009, 2009. 
[30] VAN ZIJL, G.P.A.G., BORST, R., ROTS, J.G. "The role of crack rate dependence in the long-term behaviour of cementitious materials". International Journal of Solids and Structures. v. 38, n. 30-31, pp. 5063-5079, 2001.

[31] PESCE, G.L., Study of carbonation in novel lime based materials, in Department of Architecture and Civil Engineering, University of Bath: Bath, England, 2014.

[32] CAZALLA, O., NAVARRO, C.R., SEBASTIAN, E., et al., "Aging of lime putty: Effects on traditional lime mortar carbonation”. Journal of American Ceramic Society. v. 83, n. 5, pp. 1070-1076, 2000.

[33] ELERT, K., RODRIGUEZ-NAVARRO, C., PARDO, E.S., et al., "Lime Mortars for the Conservation of Historic Buildings". International Institute for Conservation of Historic and Artistic Works. v. 47, n. 1, pp. $62-75,2002$.

[34] RODRIGUEZ-NAVARRO, C., CAZALLA, O., ELERT, K., et al., "Liesegang pattern development in carbonating traditional lime mortars". Proceeding of The Royal Society. v. 458, pp. 2261-2273, 2002.

[35] LAWRENCE, R.M., MAYS, T.J., RIGBY, S.P., et al., "Effects of carbonation on the pore structure of non-hydraulic lime mortars". Cement and Concrete Research. v. 37, n. 7, pp. 1059-1069, 2007.

[36] LAWRENCE, R.M.H., A Study of Carbonation in Non-Hydraulic Lime Mortars, in Department of Architecture and Civil Engineering, University of Bath: Bath, 2006.

[37] HOUST, Y.F., "The role of moisture in the carbonation of cementitious materials". International Journal for Restoration of Buildings Monuments. v. 2, pp. 49-66, 1996.

[38] HOUST, Y.F., SADOUKI, H., WITTMANN, F.H. Influence of aggregate concentration on the diffusion of $\mathrm{CO}_{2}$ and $\mathrm{O}_{2}$, in Interfaces in Cementitious composites, R.P.P. 18, Editor, London, etc: E \& F SPON: Toulouse, 1993.

[39] HOUST, Y.F. , WITTMANN, F.H. "Influence of porosity and water content on the diffusivity of $\mathrm{CO}_{2}$ and $\mathrm{O}_{2}$ through hydrated cement paste". Cement and Concrete Research. v. 24, n. 6, pp. 1165-1176, 1994.

[40] IZAGUIRRE, A., LANAS, J., ÁLVAREZ, J.I. "Ageing of lime mortars with admixtures: Durability and strength assessment”. Cement and Concrete Research. v. 40, n. 7, pp. 1081-1095, 2010.

[41] HENRIQUES, F.M.A., RATO, V.M., CHAROLA, A.E. The influence of grain size distribution on the performance of mortars, in Proceedings of the 10th international congress on deterioration and conservation of stone, e. D. Kwiatkowski and R. Lifvendahl, Editor: Stockholm: ICOMOS, 2004.

[42] SCANNELL, S., LAWRENCE, M., WALKER, P. "Impact of Aggregate Type on Air Lime Mortar Properties". Energy Procedia. v. 62, pp. 81-90, 2014.

[43] ARIZZI, A., MARTÍNEZ MARTÍNEZ, J., CULTRONE, G., et al., "Mechanical Evolution of Lime Mortars During the Carbonation Process". Key Engineering Materials. 465,: p. 483-486, 2011.

[44] VERSTRYNGE, E., SCHUEREMANS, L., VAN GEMERT, D., et al., "Monitoring and predicting masonry's creep failure with the acoustic emission technique". $N D T \& E$ International. v. 42, n.6, pp. 518523, 2009.

[45] GIMBERT, S.J., A Combined Empirical and Computational Approach to Creep in Replicas of Historic Mortar, Pennsylvania State University at University Park, 2008.

[46] LAWRENCE, R.M.H. A critical review of techniques used for the assessment of carbonation in lime mortars. In 2005 International Building Lime Symposium,pp. 15. Orlando, Florida, USA: 2005.

[47] LO, Y. , LEE, H.M., "Curing effects on carbonation of concrete using a phenolphthalein indicator and Fourier-transform infrared spectroscopy". Building and Environment. v. 37, n. 5, pp. 507-514, 2002.

[48] SLEGERS, P.A., ROUXHET, P.G. "Carbonation of the hydration products of tricalcium silicate". Cement and Concrete Research. v. 6, n. 3, pp. 381-388, 1976.

[49] VILLAIN, G., THIERY, M., PLATRET, G. "Measurement methods of carbonation profiles in concrete: Thermogravimetry, chemical analysis and gammadensimetry". Cement and Concrete Research. v. 37, n. 8, pp. 1182-1192, 2007.

[50] MEHTA, P., MONTEIRO, P.J.M. Concrete: Microstructure, Properties, and Materials: McGraw-Hill Education, 2005.

[51] RILEM, "CPC-18 Measurement of hardened concrete carbonation depth, TC 56-MHM". Materials and Structures. v. 21, n. 126, pp. 453 - 455, 1988.

[52] FATTUHI, N.I., "Concrete carbonation as influenced by curing regime". Cement and Concrete Research.v. 18, n. 3, pp. 426-430, 1988.

[53] HOUST, Y.F., WITTMANN, F.H. "Depth profiles of carbonates formed during natural carbonation". Cement and Concrete Research. v. 32, n. 12, pp. 1923-1930, 2002.

[54] KOBAYASHI, K., SUZUKI, K., UNO, Y. "Carbonation of concrete structures and decomposition of CS-H”. Cement and Concrete Research. v. 24, n. 1, pp. 55-61, 1994.

[55] PARROTT, L.J., KILLOH, D.C. "Carbonation in a 36 year old, in-situ concrete". Cement and Concrete Research. v. 19, n. 4, pp. 649-656, 1989. 
[5e6] GONÇALVES, A.P.A., Corrosion Prevention in Historic Concrete - Monitoring the Richards Medical Laboratories, University of Pennsylvania: Philadelphia, PA, USA, 2011.

[57] EARNEST, C.M., The modern thermogravimetric approach to the compositional analysis of materials, in Compositional analysis by Thermogravimetry, C.M. Earnest, Editor, Philadelphia: American Society for Testing and Material, 1988.

[58] ADAMS, J., DOLLIMORE, D., GRIFFITHS, D.L. "Thermal analytical investigation of unaltered $\mathrm{Ca}(\mathrm{OH})_{2}$ in dated mortars and plasters". Thermochimica Acta. v. 324, n. 1-2, pp. 67-76, 1998.

[59] ADAMS, J., KNELLER, W., DOLLIMORE, D. "Thermal analysis (TA) of lime and gypsum-based medieval mortars". Thermochimica Acta. v. 211, n. 10, pp. 93-106, 1992.

[60] CIZER, O., Competition between carbonation and hydration on the hardening of calcium hydroxide and calcium silicate binders, K. U. Leuven: Leuven, Belgium, 2009.

[61] GAMEIRO, A., SANTOS SILVA, A., FARIA, P., et al., "Physical and chemical assessment of limemetakaolin mortars: Influence of binder:aggregate ratio". Cement and Concrete Composites. v. 45, pp. 264$271,2014$.

[62] KURIYAN, J., KONFORTI, B., WEMMER, D. The Molecules of Life: Physical and Chemical Principles: Taylor \& Francis Group, 2012.

[63] LAWRENCE, R.M.H., MAYS, T.J., WALKER, P., et al., "The use of tg to measure different concentrations of lime in non-hydraulic lime mortars". Journal of Thermal Analysis and Calorimetry. v. 85, n. 2, pp. 377-382, 2006.

[64] KELTER, P.B., MOSHER, M.D., SCOTT, A. Chemistry: The Practical Science: Houghton Mifflin, 2008.

[65] ATKINS, P., Shriver and Atkins' Inorganic Chemistry. $5^{\text {th }}$ edition ed: OUP Oxford, 2010.

[66] KOTZ, J., TREICHEL, P., TOWNSEND, J. Chemistry and Chemical Reactivity, Enhanced Edition: Cengage Learning, 2009.

[67] OLIVEIRA, M.A., AZENHA, M., LOURENÇO, P.B. "Simulation of Humidity Fields in Concrete: Experimental Validation and Parameter Estimation ”. Journal of Advanced Concrete Technology - J-Stage. v. 13, n. 4, pp. 214-229, 2015.

[68] ATKINS, P. , PAULA, J. Atkins' Physical Chemistry. $9^{\text {th }}$ edition ed: OUP Oxford, 2014.

[69] BSI, BS-EN 459-1 BSI Standards Publication Building lime in Part 1: Definitions, specifications and conformity criteria, The British Standards Institution BS EN 2010.

[70] TA, TA Instruments - Thermal Solutions - User Reference Guide - Vol 1, W. Corporation, Editor: New Castle, USA, 1997.

[71] JENKINS, R., X-Ray Fluorescence Spectrometry. $2^{\text {nd }}$ edition ed: Wiley, 1999.

[72] LANGHOFF, N., SIMIONOVICI, A., ARKADIEV, V., et al., Handbook of Practical X-Ray Fluorescence Analysis, B. Beckhoff, Kanngießer, B., Langhoff, N.,Wedell, R., and Wolff, H., Editor. 2006, Springer-Verlag Berlin Heidelberg. p. 878.

[73] SILVA, D.A., WENK, H.R., MONTEIRO, P.J.M. "Comparative investigation of mortars from Roman Colosseum and cistern". Thermochimica Acta. v. 438, n. 1-2, pp. 35-40, 2005.

[74] BSI, BS-EN-13139:2002 - Aggregates for mortar, The British Standards Institution, 2002.

[75] SCHICK, H.L., "A Thermodynamic Analysis of the High-temperature Vaporization Properties of Silica". Chemical Reviews. v. 60, n. 4, pp. 331-362, 1960.

[76] CEN, CEN-EN-1015-2: Bulk sampling of mortars and preparation of ters mortars - Part 2, European Committee for Standardization, 1999.

[77] SEABRA, M.P., PAIVA, H., LABRINCHA, J.A., et al., "Admixtures effect on fresh state properties of aerial lime based mortars". Construction and Building Materials. v. 23, n. 2, pp. 1147-1153, 2009.

[78] ALGARVIO, M.P.R.P., "Influence of water/lime ratio on the characteristics of lime mortars for use as substitution renders in ancient buildings, Extended Abstract". Journal., p. 15, 2010.

[79] FORSTER, A., "Hot-Lime Mortars: A Current Perspective". Journal of Architectural Conservation. v. 10, n. 3, pp. 7-27, 2004.

[80] MALINOWSKI, E.S., HANSEN, T.S. "Hot Lime Mortar in Conservation-Repair and Replastering of the Façades of Läckö Castle". Journal of Architectural Conservation. v. 17, n. 1, pp. 95-118, 2011.

[81] MARGALHA, G., VEIGA, R., SILVA, A.S., et al., "Traditional methods of mortar preparation: The hot lime mix method". Cement and Concrete Composites. v. 33, n. 8, pp. 796-804, 2011.

[82] MOROPOULOU, A., TSIOURVA, T., BISBIKOU, K., et al., "Hot lime technology imparting high strength to historic mortars". Construction and Building Materials. v. 10, n. 2, pp. 151-159, 1996.

[83] VÁLEK, J., MATAS. T. Experimental study of hot mixed mortars in comparison with lime putty and hydrate mortars. In 2nd Conference on Historic Mortars - HMC 2010 and RILEM TC 203-RHM final workshop,pp. Prague: 2010. 
[84] VÁLEK, J. , MATAS, T. Experimental Study of Hot Mixed Mortars in Comparison with Lime Putty and Hydrate Mortars, in Historic Mortars, J. Válek, J.J. Hughes, and C.J.W.P. Groot, Editors. 2012, Springer Netherlands. pp. 269-281.

[85] CEN, CEN-EN-196-1-Methods of testing cement. Determination of strength, European Committee for Standardization, 2005.

[86] VEIGA, M.R., SOUZA, R. "Metodologia de avaliação da retraç̧ão livre das argamassas desde a sua moldagem (in Portuguese)". Revista de Engenharia Civil da Universidade do Minho. v. 20, pp. 45-56, 2004.

[87] SAETTA, A.V., SCHREFLER, B.A., VITALIANI, R.V. "The carbonation of concrete and the mechanism of moisture, heat and carbon dioxide flow through porous materials". Cement and Concrete Research. v. 23, n. 4,. pp. 761-772, 1993.

[88] SAETTA, A.V., VITALIANI, R.V. "Experimental investigation and numerical modeling of carbonation process in reinforced concrete structures: Part I: Theoretical formulation". Cement and Concrete Research. v. 34, n. 4, pp. 571-579, 2004.

[89] SAETTA, A.V., VITALIANI, R.V. "Experimental investigation and numerical modeling of carbonation process in reinforced concrete structures: Part II. Practical applications". Cement and Concrete Research. v. 35, n. 5, pp. 958-967, 2005.

[90] OLIVEIRA, M., AZENHA, M., LOURENÇO, P.B., et al., "Estudo experimental do fluxo de umidade em argamassas de cal aérea". Revista Matéria. v. 24, n. 12, pp. 14, 2019.

[91] PAPADAKIS, V., VAYENAS, C.G., FARDIS, M.N. "Fundamental modeling and experimental investigation of concrete carbonation". ACI Materials Journal. v. 88, n. 4, pp. 363-373, 1991.

[92] PAPADAKIS, V.G., MICHAEL, N.F., et al. "Fundamental Modeling and Experimental Investigation of Concrete Carbonation". Materials Journal. v. 88, n. 4, pp. 363-373, 1991.

[93] SAETTA, A.V., SCOTTA, R.V., VITALIANI, R.V. "Analysis of Chloride Diffusion into Partially Saturated Concrete". Materials Journal. v. 90, n.5, pp. 441-451, 1993.

[94] SAETTA, A.V., SCHREFLER, B.A., VITALIANI, R.V.. "2-D model for carbonation and moisture/heat flow in porous materials". Cement and Concrete Research. v. 25, n. 8, pp. 1703-1712, 1995.

[95] LIETH, R.M.A., Preparation and Crystal Growth of Materials with Layered Structures: Springer Netherlands, 2013.

[96] CIZER, O., VAN BALEN. K., VAN GEMERT, D. Carbonation reaction of lime hydrate and hydraulic binders at $20^{\circ} \mathrm{C}$, in First International Conference on Accelerated Carbonation for Environmental and Materials, T.R. Society, Editor: London, UK, 2006

[97] LACKNER, K.S., "Carbonate Chemistry for Sequestering Fossil Carbon". Annual Review of Energy and the Environment. 27, pp. 193-232, 2002.

[98] AL-BASHAIREH, K.S., Chronology and technological production styles of Nabatean and Roman plasters and mortars at Petra (Jordan), The University of Arizona, 2008.

[99] DANIELE, V., TAGLIERI, G. $\mathrm{Ca}(\mathrm{OH})_{2}$ Nanoparticle Characterization: Microscopic Investigation Of Their Application On Natural Stones, in Materials Characterisation V, C.A.B. A. A. Mammoli, and A. Klemm, Editor: Valencia, Spain, 2015.

[100] MONTES-HERNANDEZ, G., CHIRIAC, R., TOCHE, F., et al., "Gas-solid carbonation of $\mathrm{Ca}(\mathrm{OH})_{2}$ and $\mathrm{CaO}$ particles under non-isothermal and isothermal conditions by using a thermogravimetric analyzer: Implications for $\mathrm{CO}_{2}$ capture". International Journal of Greenhouse Gas Control. v. 11, pp. 172-180, 2012.

[101] FERNANDEZBERTOS, M., SIMONS, S., HILLS, C. et al., "A review of accelerated carbonation technology in the treatment of cement-based materials and sequestration of $\mathrm{CO}_{2}$ ". Journal of Hazardous Materials. v. 112, n. 3, pp. 193-205, 2004.

[102] ROUCHON, L., FAVERGEON, L., PIJOLAT, M. "Analysis of the kinetic slowing down during carbonation of $\mathrm{CaO}$ by CO2". Journal of Thermal Analysis and Calorimetry. v. 113, n. 3, pp. 1145-1155, 2013.

[103] MONTES-HERNANDEZ, G., DAVAL, D., CHIRIAC, R., et al., "Growth of Nanosized Calcite through Gas-Solid Carbonation of Nanosized Portlandite under Anisobaric Conditions". Crystal Growth \& Design. v. 10, n. 11, pp. 4823-4830, 2010.

[104] MONTES-HERNANDEZ, G., A. POMMEROL, F. RENARD, et al., "In situ kinetic measurements of gas-solid carbonation of $\mathrm{Ca}(\mathrm{OH})_{2}$ by using an infrared microscope coupled to a reaction cell". Chemical Engineering Journal. v. 161, n. 1-2, pp. 250-256, 2010.

[105] SEREDA, P.J., FELDMAN, R.F., SWENSON. E.G. Effect of sorbed water on some mechanical properties of hydrated Portland cement pastes and compacts. In Proceedings of Symposium on Structure of Portland Cement Paste and Concrete, Highway Research Board,pp. 58-73. . Washington D. C.: 1966.

[106] CAZALlA, O., C. NAVARRO, S. R., E.,, et al., " Aging of lime putty: Effects on traditional lime mortar carbonation". Journal of American Ceramic Society. 83: p. 1070-1076, 2000. 


\section{ORCID}

Mateus A. N. Oliveira

https://orcid.org/0000-0002-3027-3312

Miguel Azenha

https://orcid.org/0000-0003-1374-9427

Paulo Barbosa Lourenço

https://orcid.org/0000-0001-8459-0199

José Victor B. Souza

https://orcid.org/0000-0001-5274-0973 